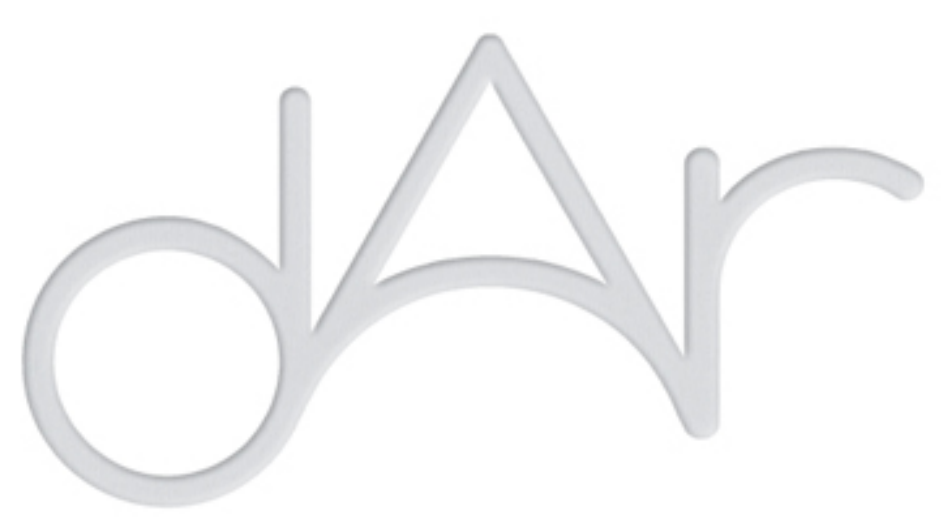

\title{
Análisis espectroscópicos de restos arqueológicos provenientes de yacimientos romanos de medellín (badajoz) y su entorno
}

\author{
Autor(es): $\quad$ Nuevo, M. J.; Sánchez, A. Martín; Ojeda, M. A.; Millán, S. Guerra \\ Publicado por: Imprensa da Universidade de Coimbra \\ URL \\ persistente: $\quad$ URI:http://hdl.handle.net/10316.2/42871 \\ DOI: $\quad$ DOI:https://doi.org/10.14195/2182-844X_3_12 \\ Accessed : $\quad$ 26-Apr-2023 15:02:10
}

A navegação consulta e descarregamento dos títulos inseridos nas Bibliotecas Digitais UC Digitalis, UC Pombalina e UC Impactum, pressupõem a aceitação plena e sem reservas dos Termos e Condições de Uso destas Bibliotecas Digitais, disponíveis em https://digitalis.uc.pt/pt-pt/termos.

Conforme exposto nos referidos Termos e Condições de Uso, o descarregamento de títulos de acesso restrito requer uma licença válida de autorização devendo o utilizador aceder ao(s) documento(s) a partir de um endereço de IP da instituição detentora da supramencionada licença.

Ao utilizador é apenas permitido o descarregamento para uso pessoal, pelo que o emprego do(s) título(s) descarregado(s) para outro fim, designadamente comercial, carece de autorização do respetivo autor ou editor da obra.

Na medida em que todas as obras da UC Digitalis se encontram protegidas pelo Código do Direito de Autor e Direitos Conexos e demais legislação aplicável, toda a cópia, parcial ou total, deste documento, nos casos em que é legalmente admitida, deverá conter ou fazer-se acompanhar por este aviso.

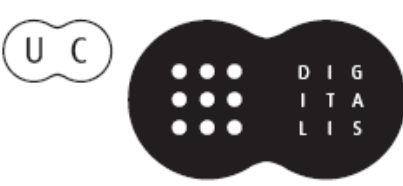




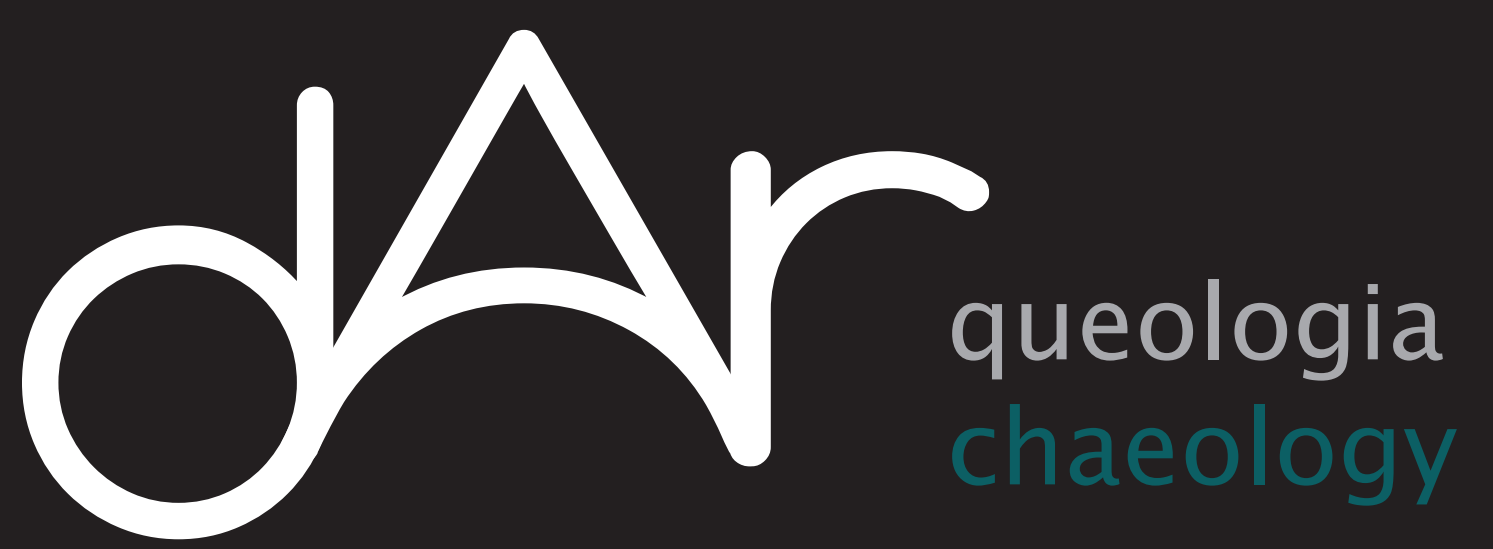

q

ig

u

논

e s

it<smiles>c1ccccc1</smiles>

$\mathrm{S}$

al $t \mathrm{t}$

e e

C C

t $\mathrm{t}$

u

c

\section{chaeology}

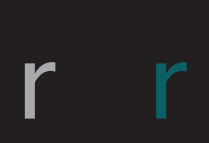

a e

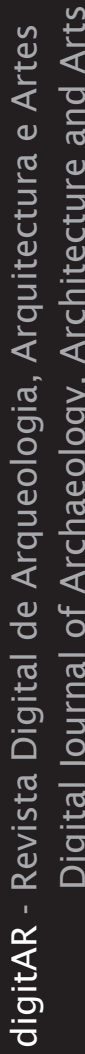




\section{ANÁLISIS ESPECTROSCÓPICOS DE RESTOS ARQUEOLÓGICOS PROVENIENTES DE YACIMIENTOS ROMANOS DE MEDELLÍN (BADAJOZ) \\ Y SU ENTORNO.}

\section{SPECTROSCOPIC ANALYSIS OF ARCHAEOLOGICAL REMAINS FROM ROMAN SITES OF MEDELLÍN (BADAJOZ) AND ITS ENVIRONMENT}

\section{J. Nuevo* \\ Departamento de Física, Universidad de Extremadura, 06006 Badajoz, España}

\section{A. Martín Sánchez** \\ Centro de Conservación y Restauración de Bienes Culturales, Dirección General de Patrimonio Cultural, Consejería de Educación y Cultura, Gobierno de Extremadura, 06800 Mérida, España}

\author{
M. A. Ojeda*** \\ Sección de Arqueología, Dirección General de \\ Patrimonio Cultural, Consejería de Educación y Cultura, \\ Gobierno de Extremadura, 06800 Mérida, España

\section{S. Guerra Millán***} \\ Sección de Arqueología, Dirección General de \\ Patrimonio Cultural, Consejería de Educación y Cultura, \\ Gobierno de Extremadura, 06800 Mérida, España
}

\section{RESUMEN}

En este trabajo se presentan los resultados obtenidos utilizando diversas técnicas espectrométricas que han permitido la caracterización de vestigios romanos, islámicos y modernos encontrados en el área de la Colonia Metellinensis, localizada en el actual Medellín (Badajoz, España). Además, se han analizado algunos restos pictóricos encontrados en la villa romana de La Majona, situada en el entorno, a unos pocos kilómetros de la Colonia, y que constituye uno de los yacimientos romanos más importantes de esa zona. Las técnicas utilizadas han sido fluorescencia de rayos $X$ (EDXRF), realizada in situ, además de difracción de rayos $X(X R D)$ y microscopía electrónica (SEM-EDX), siendo estas últimas técnicas facilitadas por los Servicios de Apoyo a la Investigación de la Universidad de Extremadura.

Palabras Clave: In situ EDXRF, XRD, SEM-EDX

\author{
ABSTRACT \\ In this work, the results obtained using \\ several spectrometric techniques allowing \\ the characterization of Roman, Islamic and \\ modern vestiges found in the area of the \\ Colonia Metellinensis, in the modern village \\ of Medellin (Badajoz, Spain), are presented. \\ Moreover, several pictorial rests found in La \\ Majona Roman villa, situated in the surroundings, \\ some kilometers from the Colonia, and what \\ constitutes one of the archeological roman \\ sites most important in the area, were also \\ analyzed. The used techniques were X-ray \\ fluorescence (EDXRF), carried out in situ, X-ray \\ diffraction (XRD), and Scanning electron \\ microscopy (SEM-EDX), being the third ones \\ carried out at the facilities of the support \\ research of University of Extremadura.
}

Key words: In situ EDXRF, XRD, SEM-EDX 


\section{INTRODUCCIÓN}

La villa de Medellín (Badajoz) y su entorno constituyen un emplazamiento arqueológico de primera magnitud, donde cada día se descubren valiosos vestigios pertenecientes a todas las épocas. Es uno de los enclaves arqueológicos más antiguos de Extremadura, aunque fue en la etapa romana cuando este lugar desarrolló un notable urbanismo (Guerra Milán et al 2004), alcanzando la categoría de Colonia (Colonia Metellinensis).

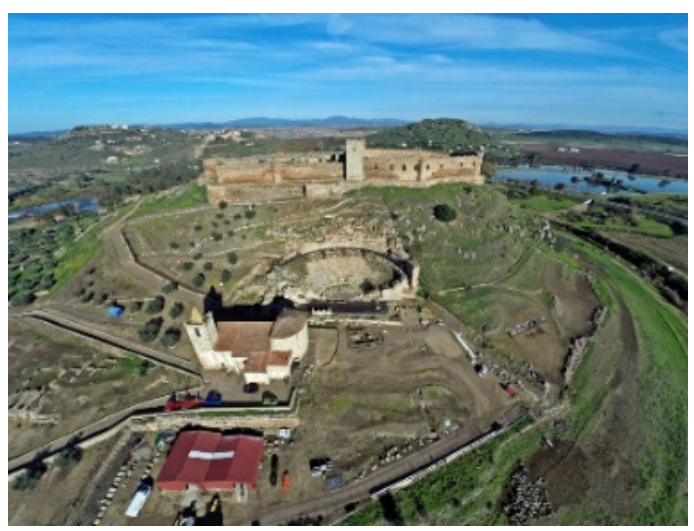

Figura 1- Vista aérea del yacimiento de Medellín, con el teatro romano en el centro

El Teatro Romano de Medellín se localiza en la ladera sur del denominado "Cerro del Castillo". Fue construido en el cambio de Era (Mateos et al 2011) siguiendo esquemas vitruvianos y conservando la tipología constructiva de los teatros griegos, erigidos sobre laderas de colinas. Gracias a la excavación arqueológica reanudada en 2007, ciertas partes de este teatro (Proedria, Orchestra, Frons Pulpiti, Scaenae Frons, entre otras), han emergido en un estado notable de conservación. En particular, varios fragmentos decorados del frente escénico (Scaenae Frons) se han recuperado en excelentes condiciones. Además, de los innumerables restos romanos pertenecientes al teatro (columnas, esculturas, etc.), en este enclave arqueológico (Figura 1), en cuya cima se levanta un castillo medieval, se han descubierto vestigios pertenecientes a otras épocas y culturas, tales como la islámica, cristiana y moderna, considerados también en este trabajo. Por otro lado y simultáneamente a los trabajos arqueológicos mencionados, se ha intervenido en otros yacimientos del entorno, como es el caso de la Villa Romana de La Majona. Ésta se sitúa a pocos kilómetros del yacimiento del Cerro del Castillo pero ya en el término municipal de Don Benito. Allí se han descubierto restos de pinturas polícromas en sus muros, además de magníficos mosaicos, lo que la convierte en uno de los yacimientos romanos más importantes de la Comarca Vegas Altas del Guadiana. Son numerosos los restos romanos tanto públicos como privados (teatros, anfiteatros, circos, villas, etc.) que se han localizado a lo largo de la geografía mundial ( Baraldi et al 2006 e Westlake et al 2012) y en la Península lbérica en particular (Villar et al 2005), aunque en este último caso, las pinturas que han sobrevivido hasta nuestra época son de restringida calidad pictórica (Durán Benito et all 2008), lo que ha limitado el análisis científico de las mismas. Aun así, destacan en ese sentido algunos estudios realizados sobre yacimientos de entornos cercanos a los considerados en este trabajo, por ejemplo, los restos analizados por Edreira et al. (2001 e 2003) de los muros romanos de la Cripta del Museo y de la Alcazaba en la ciudad de Mérida, situada a 40 $\mathrm{km}$ al oeste de Medellín. Por otro lado, Hunt Ortiz et al. (2008) caracterizaron los vestigios hallados en dos yacimientos de la ciudad de Sevilla, situada a unos 200 $\mathrm{km}$ al sur de la localidad de Medellín. En este trabajo se han realizado análisis in situ de restos de pigmentos encontrados en muros, columnas y otros elementos ornamentales, mediante un equipo portátil de fluorescencia de rayos $X$ dispersiva en energía (EDXRF). Además, con objeto de completar la información obtenida mediante esta técnica elemental, parte de estas muestras junto con otras adicionales pertenecientes a estos yacimientos $y$ depositadas actualmente en el Museo Arqueológico de Badajoz, se han analizado mediante difracción de rayos $X$ 
(XRD) y microscopía electrónica (SEM), en los servicios de apoyo a la investigación de la Universidad de Extremadura (SAIUEX). Previamente a estos análisis, se realizaron cortes estratigráficos que fueron embutidos en resina y examinados al microscopio óptico en el Centro de Conservación y Restauración de la Junta de Extremadura.

\section{MATERIALES Y MÉTODOS}

a) Enclaves arqueológicos y muestras analizadas

Tres son los enclaves arqueológicos considerados en este trabajo: El Teatro Romano (TRM), La Iglesia de Santa María del Castillo, que en la sigla del material recuperado en su excavación se denominó como Iglesia del castillo (ICM) y La Villa Romana La Majona (VRLM) (Figura 2). Los dos primeros están situados en el denominado "Cerro del Castillo", al lado de la villa actual de Medellín, encontrándose el tercero a unos $10 \mathrm{~km}$ al noreste de los anteriores. La caracterización de los vestigios pertenecientes a estos enclaves se ha realizado mediante dos tipos de estudio. Una primera etapa consistió en un análisis in situ de los pigmentos pertenecientes a elementos decorativos, tanto del Teatro (frente escénico, columnas, etc.), como de los muros de la Villa Romana (Figura 3). Estos análisis se realizaron con un equipo portátil de fluorescencia de Rayos X, del Departamento de Física de la Universidad de Extremadura. Una segunda fase de este estudio ha consistido en la caracterización de alguna de las muestras consideradas en la etapa anterior junto con otras adicionales pertenecientes a estos yacimientos pero contextualizadas en épocas diferentes, con objeto conocer la variación, tanto en los pigmentos y materiales utilizados, como en las técnicas pictóricas aplicadas. En la Figura 4 se presentan algunas de las muestras analizadas y detalladas en la Tabla 1. A este grupo de muestras, además de la fluorescencia se le aplicó la microscopía electrónica y la difracción de rayos $X$ con el objeto de conocer la estructura y la composición tanto de la capa pictórica como del substrato.
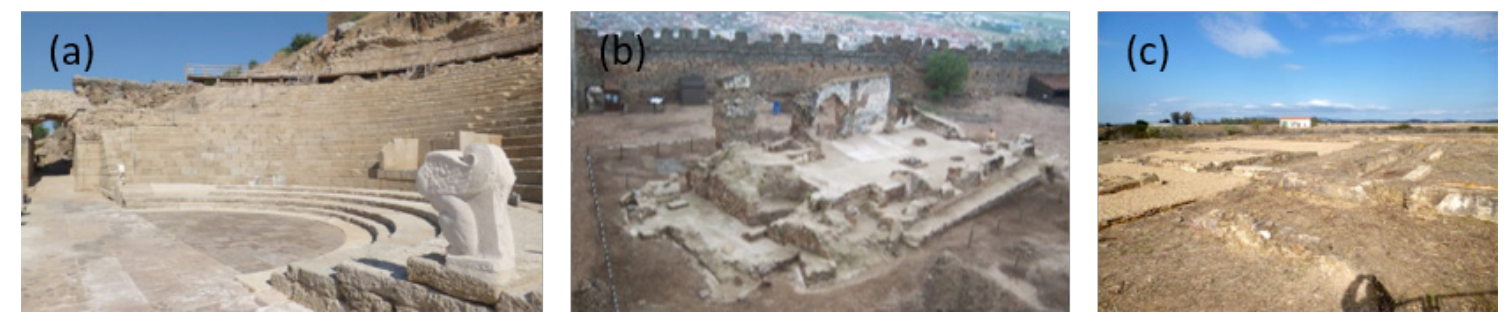

Figura 2- Los tres enclaves arqueológicos considerado en este trabajo: (a) Teatro Romano de Medellín (TRM), (b) Iglesia de Santa María del Castillo (ICM) y (c) Villa Romana La Majona (VRLM).
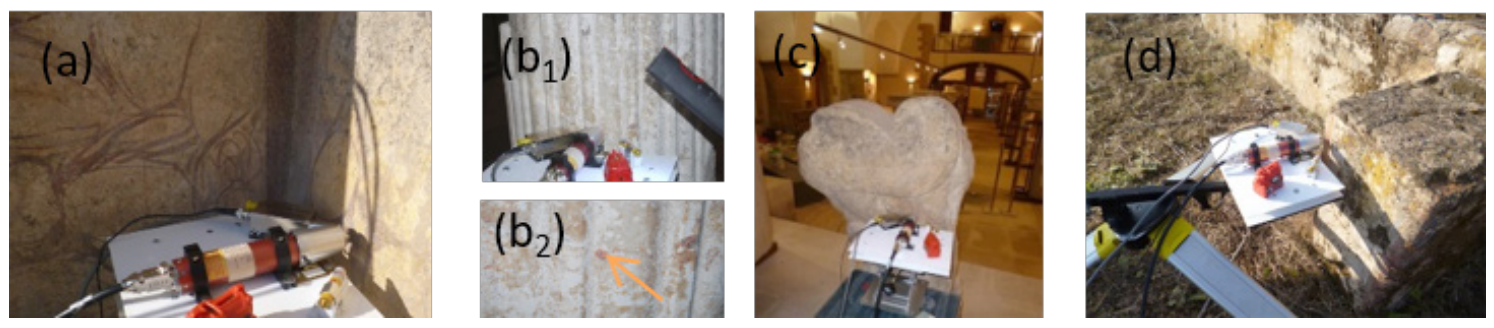

Figura 3- Elementos decorativos del TRM (a-c) y VRLM (d) analizados mediante fluorescencia de rayos X: (a) Frente escénico, (b) columna, (c) esfinge y (d) muros. 

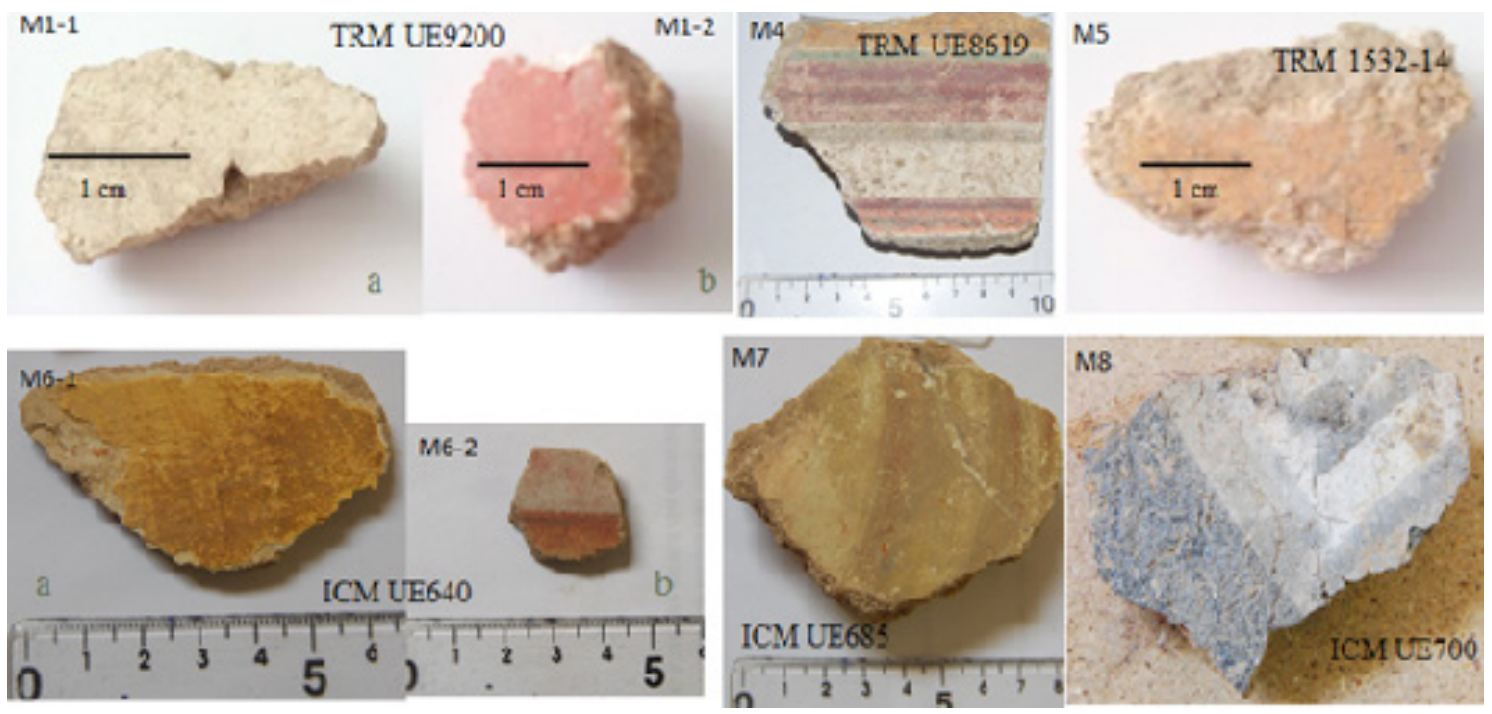

Figura 4- Muestras consideradas en la segunda etapa de este trabajo y descritas en la Tabla 1.

b) Técnicas de análisis

El equipo de fluorescencia está compuesto por un tubo de rayos $X$ (con ánodo de Ag, $30 \mathrm{kV}, 100 \mu \mathrm{A})$, un detector con resolución en energía de $220 \mathrm{eV}$ (FWHM @5.9keV), los dos de Amptek (Amptek, Inc., Bedford, MA) y un sistema de láseres cruzados para enfocar con precisión el punto de la muestra considerado (Nuevo et al 2011). Los ensayos ex situ, se llevaron a cabo en el SIAUEX. LOs análisis de microscopía electrónica se realizaron en un Quanta 3D FEl (FEl Company II) de barrido en modo de bajo vacío, con dos tipos de detectores, de electrones secundarios y retrodispersados. Para este propósito se utilizaron cortes estratigráficos de las muestras embutidas en resina, observadas y fotografiadas previamente en un microscopio óptico (x40). Por último, los análisis de difracción se realizaron en un D8 ADVANCE de Bruker en dos modos

Tabla 1: Relación de muestras utilizadas en este trabajo referenciadas a la excavación en cada caso. Las muestras M1 a M5 corresponden a muestras romanas pertenecientes al Teatro Romano de Medellín (TRM) mientras que la M6 (moderna), M7 (cristiana) y M8 (islámica) provienen de la Iglesia del Castillo de Medellín (ICM).

\begin{tabular}{|c|c|c|c|c|c|}
\hline TRM & ref. excavaciór & n color & ICM & ref. excavación & color \\
\hline M1-1 & UE9200 & Blanco & M6-1 & UE640 (moderna s. XVI-XVIII) & Amarillo \\
\hline M1-2 & “ & Rojo & M6-2 & “ & $\begin{array}{r}\text { Rojo } \\
\text { Blanco }\end{array}$ \\
\hline M2 & UE9070 & Rojo & & & \\
\hline M3 & UE9089 & Blanco & M7 & UE685 (cristiana s. XIII?) & Gris verdoso \\
\hline M4 & UE8619 & $\begin{array}{l}\text { Naranja } \\
\text { Verde } \\
\text { Blanco } \\
\text { Rojo }\end{array}$ & M8 & UE700 (islámica s. XII-XIII?) & $\begin{array}{l}\text { Blanco } \\
\text { Negro }\end{array}$ \\
\hline M5 & $1532-14$ & Ocre & & & \\
\hline
\end{tabular}


diferentes: incidencia rasante (con ángulo de incidencia fijo entre uno y dos grados) e incidencia acoplada (con variación del ángulo de incidencia entre 10 y $80^{\circ}$ ), con el objeto de separar la composición de la capa pictórica respecto a la del substrato, sin tratamiento alguno de las muestras.

\section{RESULTADOS Y DISCUSIÓN}

En la Figura 3 se presentan los elementos decorativos (frente escénico, columnas, muros, etc.) analizados in situ en la primera etapa de este trabajo. Los colores presentes en estos ornamentos son blanco, rojo y ocre. Los elementos identificados en todos los casos fueron calcio (Ca), hierro (Fe) y potasio (K), con trazas de silicio (Si), titanio (Ti) y manganeso (Mn). Estos resultados sugieren la utilización de calcita en los estucos y óxidos de hierro en los pigmentos, tanto en los ocres como en los rojos. La ausencia de mercurio $(\mathrm{Hg})$ nos indica que, a pesar de la cercanía de las minas de Almadén (Ciudad Real), el cinabrio no fue aplicado en ningún caso. De las muestras referenciadas en la Tabla 1 y mostradas en la Figura 4, la M4, M6-1, M6-2, M7 y $M 8$ fueron analizadas in situ por EDXRF en el Museo Arqueológico Provincial de Badajoz que es dónde están actualmente depositados dichos materiales. De algunas de ellas se tomaron pequeñas lascas transversales para, una vez embutidas en resina, poder estudiar su estratigrafía. Los análisis de fluorescencia realizados sobre las capas pictóricas, identificaron al Ca y al Fe como elementos destacados, con trazas de Mn y Ti en las todas las muestras y en todos los colores, excepto en el color negro de la M8 (islámica), donde el Ca no está presente. Un resultado análogo se obtiene al analizar los morteros, donde de nuevo Ca y Fe son los elementos predominantes en todas las muestras excepto en la M8 (Figura 5). Este resultado corroboraría la inspección visual de que se tratase de un mortero de adobe. Lo más destacable en estos análisis fue la identificación de plomo (Pb), en la franja naranja de la muestra M4 (romana), que sugiere la presencia de minio (Pb3O4) como causante de esa tonalidad.

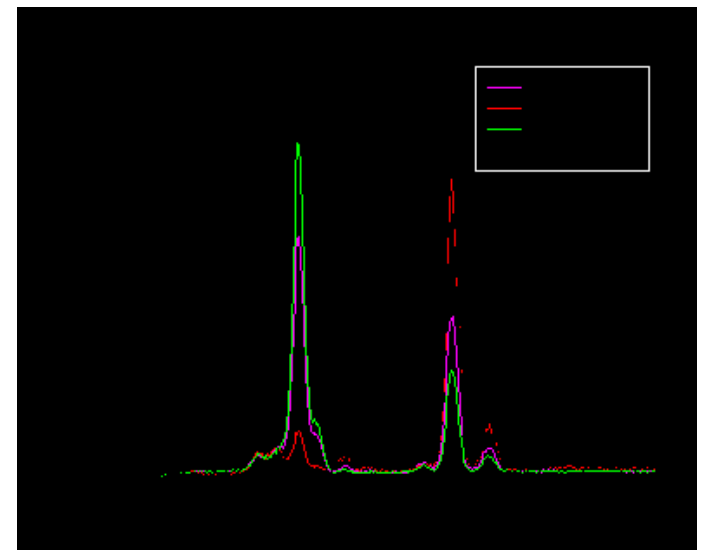

Figura 5- Comparación de los espectros de fluorescencia correspondientes a los morteros de las muestras M6 (moderna), M7 (cristiana) y M8 (islámica), pertenecientes a la iglesia del castillo (ICM).

Los análisis de difracción aplicados a las muestras M1-1, M1-2, M2, M3 y M5, confirman la presencia de calcita para los blancos y goethita, magnetita y hematites en los rojos y ocres (Figura 6). La existencia en todas las muestras analizadas de cuarzo y calcita indica que pertenecen al substrato, aunque este último componente sea también el responsable del color blanco.

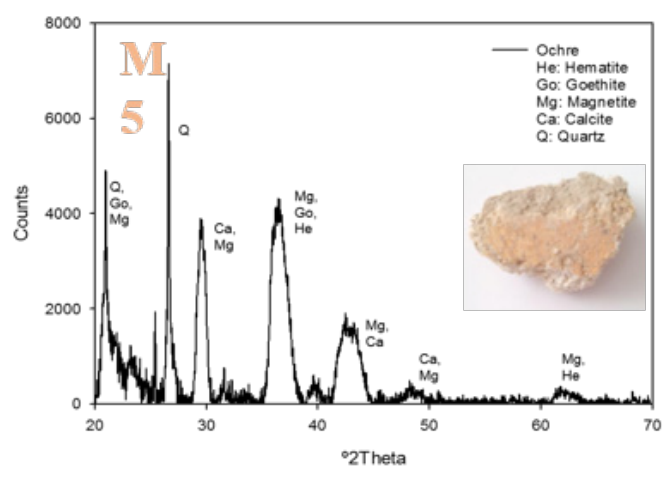

Figura 6- Difractograma de la muestra M5, perteneciente a una columna del Teatro Romano de Medellín y conservada en el centro de interpretación.

Con objeto de conocer la estructura de capas y del sustrato, los cortes 
estratigráficos fueron analizados mediante microscopía electrónica (SEM), previamente observados al microscopio óptico (Figura 7). En cuanto a las muestras romanas se refiere (M1-1, M1-2, M4, M5), se observa una gran similitud en los morteros, presentando forma y granulometría equivalente. Estos morteros están cubiertos, excepto en la muestra M1-2, por una fina capa de extraordinaria lisura (observada incluso a ojo desnudo), indicando un pulimiento de la misma. Esta fina capa que en la muestra M5 (Figura
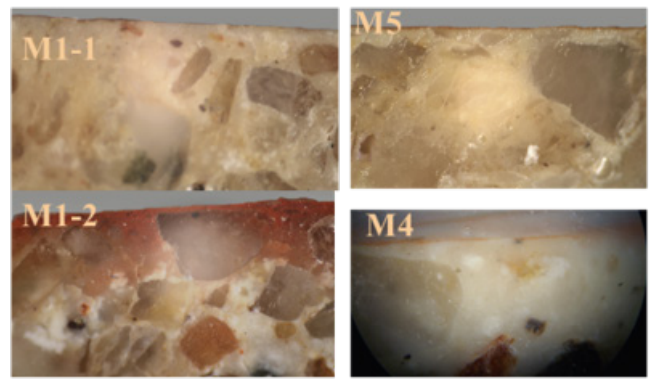

8) tiene un espesor medio de, está tan pulida que evita la impregnación de pintura en el substrato. Esto sugiere que la técnica de aplicación de la pintura no pudo ser al fresco, y señala el uso de alguna sustancia impermeabilizante entre el mortero y la capa pictórica. De no ser así, aun cuando la técnica no hubiera sido al fresco, la pintura habría penetrado algo en el mortero (la pintura, sea de la naturaleza que sea, siempre se aplica líquida, y por lo tanto mancha la capa subyacente si ésta es porosa).
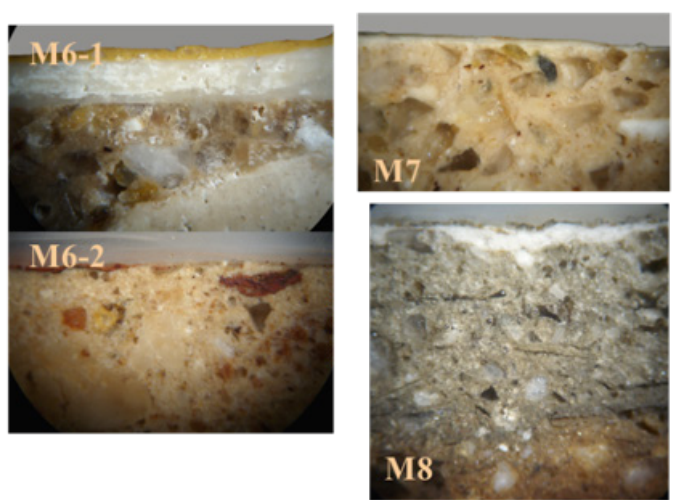

Figura 7- Cortes estratigráficos observados al microscopio óptico (x40).

El registro elemental sobre esta zona señalada en la Figura 8 presenta otros elementos tales como aluminio (AI), oxígeno (O), y fósforo (P), no identificados en los análisis de fluorescencia. La aparente discrepancia es debida a la deficiente eficiencia de los equipos de fluorescencia en la zona de bajas energías (). La presencia de Al y Si puede deberse a la utilización de caolinita (AI2Si2O5(OH)4), junto con hematites. Esta hipótesis, que necesita ser corroborada con experimentos adicionales, es plausible debido a la existencia de depósitos de este compuesto en el entorno [12].
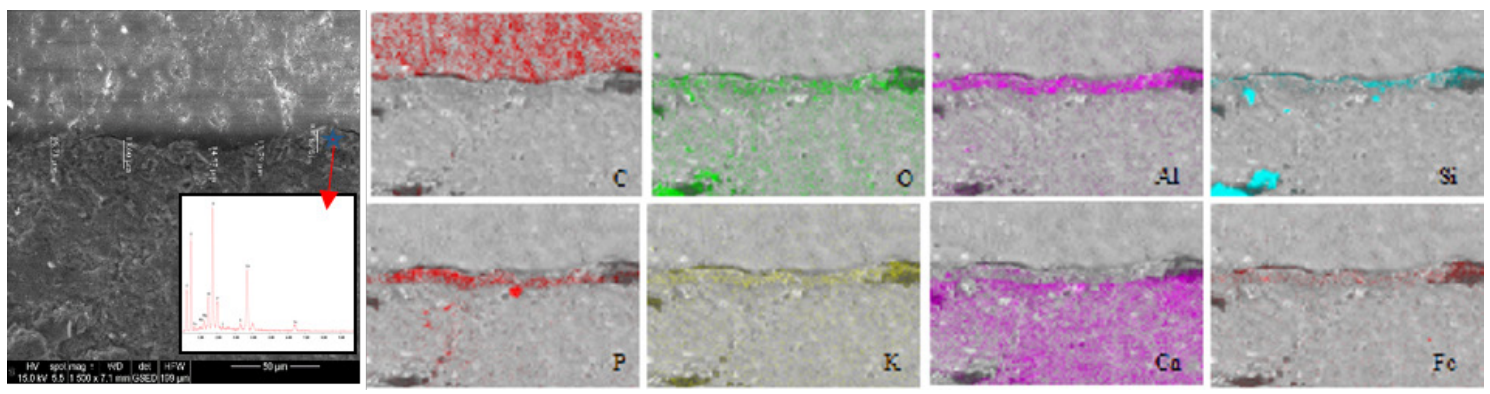

Figura 8- Imagen SEM-EDX de la muestra M5 (romana) con el mapeo elemental de la zona analizada, donde se aprecia la capa pictórica con un espesor medio de. 
Por otro lado, la observación de la estratigrafía del resto de muestras nos indica la utilización de materiales y técnicas diferentes en cada una de ellas. Por una parte, nos encontramos con las muestras M6-1 y M6-2 que, aunque pertenecen a la misma cronología (moderna), nada tienen en común entre ellas. Mientras en la M6-1 se aprecian dos capas pictóricas gruesas bien diferenciadas, sobre un mortero de granulometría gruesa, la muestra M6-2 exhibe una granulometría fina en el mortero, culminado por una capa pictórica también fina. Las capas pictóricas de estas muestras presentan una superposición de estratos con espesores irregulares, como puede comprobarse en el análisis SEM en la Figura 9. Para su comparación, se han incluido las imágenes correspondientes a la
M6-2 y M7. Sin embargo, la diferencia más destacada tanto en la estructura como en la composición, está en la muestra de procedencia islámica (M8). Esta muestra consta de dos capas muy porosas de mortero de adobe y arena, la inferior marrón y la superior negruzca, culminadas por una fina capa de pintura blanca y negra con restos de lo que parecen ser motivos geométricos. Los análisis realizados mediante SEM-EDX en la intersección de estas dos capas, sin embargo, no muestran diferencias apreciables en la composición elemental, tratándose en ambos casos de tierras arcillosas cuya coloración está relacionada con el grado de impurezas que contiene. La tonalidad más parduzca sugiere un mayor contenido en Fe, aunque este término no se ha reflejado en los restantes análisis realizados.
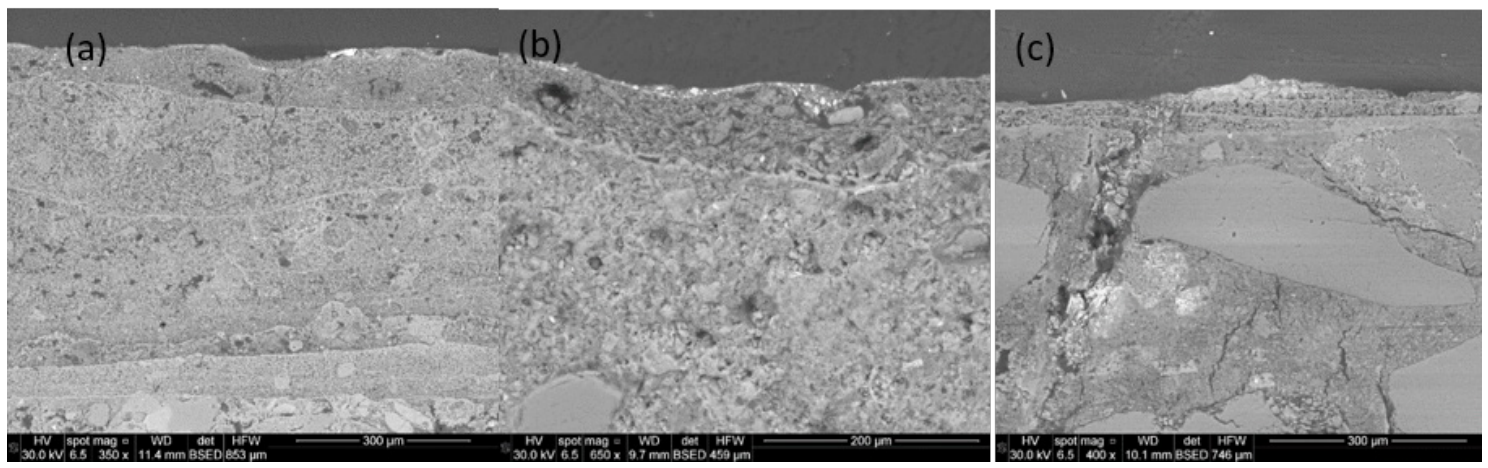

Figura 9- Imagen SEM de las muestras a) M6-1, b) M6-2 y C) M7, donde se aprecia la estructura de capas irregulares en el primer caso, frente a las otros dos.

\section{CONCLUSIONES}

En este estudio se han analizado vestigios pertenecientes a tres yacimientos arqueológicos: TRM, ICM y VRLM. La identificación mediante EDXRF de hierro y calcio como elementos mayoritarios en los pigmentos de los muros y elementos decorativos de los enclaves TRM y VRLM, sugiere la utilización de calcita y óxidos de hierro como causantes de su coloración. Además, los análisis de difracción han determinado la presencia de calcita y cuarzo en todos los casos, lo que sugiere que estos compuestos sean los responsables de los morteros. Estos análisis han identificado hematites, goethita y magnetita en los pigmentos ocres y rojos de las muestras romanas. Por otra parte, mediante cortes estratigráficos, se han podido diferenciar los morteros y capas pictóricas de las distintas muestras. Los fragmentos pertenecientes al emplazamiento de la Iglesia de Santa María del Castillo (ICM), presentan notables diferencias respecto a los restos romanos. Algunos de los restos pictóricos recuperados en el desescombro de esta Iglesia pertenecen a las pinturas 
que decoraban el interior de este templo pero hay otras, realizadas sobre adobe, que se localizan bajo los suelos de dicha Iglesia qué, según los datos obtenidos en esa intervención arqueológica, parecen ser parte de un edificio musulmán anterior que posiblemente se destruyera para construir encima la Iglesia de Santa María ya citada. Así, mientras que los morteros romanos, en general, exhiben semejanza en textura, color y granulometría, con una técnica tan depurada en las superficies que las hace prácticamente impermeables, los restantes morteros no presentan apenas características en común, siendo la de cronología islámica la que exhibe una estructura y composición completamente diferentes al resto. En este caso se trata de un mortero de adobe rico en hierro, con ausencia de calcio, y con un posible uso de caolín para el color blanco.

\section{AGRADECIMIENTOS}

Los autores agradecen al Servicio de Análisis y Caracterización de Sólidos y Superficies de los SAIUEx (financiados por UEX, Junta de Extremadura, MICINN, FEDER y FSE) el apoyo técnico y humano, y al Gobierno de Extremadura el apoyo económico (GR10160, cofinanciado con FEDER). Además, agradecemos el apoyo prestado desde la Dirección General de Patrimonio Cultural y desde el Museo Arqueológico Provincial de Badajoz para poder llevar a cabo los análisis de los materiales que se recogen en este trabajo.

\section{BIBLIOGRAFIA}

Baraldi, P., Bonazzi, A., Giordani, N., Paccagnella F. \& Zannini, P. 2006. "Analytical characterization of Roman plasters of the 'Domus Farini', Modena". Archaeometry 48. 481-499.

Durán Benito, A., Millán Sañudo, E., Jiménez de Haro, M.C., Cárceles Pascual, J.F., Erbez, A.J. \& Pérez-Rodriguez, J.L. 2008. "Estudio técnico de la pintura parietal romana. Análisis de fragmentos provenientes de villa dei papiri (Herculano) y del jardín de la casa del bracciale d'oro (Pompeya)". Actas del VII Congreso Ibérico de Arqueometría. 680-687.

Edreira, M.C., Feliu, M.J., Fernández-Lorenzo, C. \& Martín, J. 2001. «Roman wall paintings characterization from Cripta del Museo and
Alcazaba in Mérida (Spain): chromatic, energy dispersive X-ray fluorescence spectroscopy, X-ray diffraction and Fourier transform infrared spectroscopic analysis". Anal. Chim. Acta 434. 331-345.

Edreira, M.C., Feliu, M.J., Fernández-Lorenzo, C. \& Martín, J. 2003. "Spectroscopic analysis of roman wall paintings from Casa del Mitreo in Emerita Augusta, Mérida, Spain". Talanta 59. 1117-1139

Fermo, P., Piazzalunga, A., De Vos, M. \& Andreoli M. 2013. "A multi-analytical approach for the study of the pigments used in the wall paintings from a building complex on the Caelian Hill (Rome)". Appl. Phys. A 113. 1109 1119.

Galán Hurtado, E. Martín Vivaldi, J.L. 1974. Bol. Soc. Esp. Ceram. Vidr. 13. 523-546.

Guerra Millán, S., Collado Giraldo, H., Pérez Romero, S. \& Viola Nevado, M., 2014. "Metellinum: síntesis histórica y novedades arqueológicas de esta ciudad romana". Studia Lusitania: Ciudades romanas en Extremadura 8. 195.

Hunt Ortiz, M.A., Gómez Morón, A., Ontalba Salamanca, M. A., Gómez Tubío, B., Ortega Feliu, I., García Rivero, D. \& Vázquez Paz, J. 2008. "Caracterización de la composición y estructura de pigmentos decorativos de épocas romana, islámica y moderna mediante técnicas arqueométricas". Actas del VII Congreso Ibérico de Arqueometría. 688-699.

Mateos, P. \& Picado, Y. 2011 . "El Teatro Romano de Metellinum", Madrider Mitteilungen 52. 400.

Nuevo, M. J. \& Martín Sánchez A. 2011 . Appl. Rad. and Isotop. 69, 574-79.

Villar S. E. J. \& Edwards H. G. M. 2005. "An extensive colour palette in Roman villas in Burgos, Northern Spain: a Raman spectroscopic analysis". Anal. Bioanal. Chem. 382. 283-289.

Westlake, P., Siozos, P., Philippidis, A., Apostolaki, C., Derham, B., Terlixi, A., Perdikatsis, A., Jones, R. \& D. Anglos. 2012. "Studying pigments on painted plaster in Minoan, Roman and Early Byzantine Crete. A multi-analytical technique approach". Anal. Bioanal. Chem. 402. 14131432. 